\title{
Non Minimum Phase Channel Estimation by Blind and Adaptive algorithms
}

\author{
Mohammed Zidane, Said Safi, Mohamed Sabri, Ahmed Boumezzough
}

\begin{abstract}
In this paper the problem of the Non Minimum Phase (NMP) channel identification using blind and adaptive algorithms is theoretically and numerically evaluated in noise environment case for different signal to noise ratio (SNR). For this problem, three blind algorithms based on Higher Order Cumulants (HOC) versus adaptive algorithms, i.e. Recursive Least Squares (RLS) and Least Mean Squares (LMS) are presented. In order to assess the performance of these approaches to identify the parameters of non minimum phase channels, we have selected the Macchi channel model. The simulation results in noisy environment and for different data input channel, provided to illustrate the performance of the blind approaches and compare them with adaptive algorithms.
\end{abstract}

Keywords-Higher Order Cumulants, channel identification, blind algorithms, adaptive algorithms, RLS, LMS.

\section{INTRODUCTION}

$\mathbf{R}$ ECENTLY blind channel identification has drawn a great deal of attentions in the literature [1-10], [12-15]. In this work, identification of non minimum phase channel driven by a non gaussian in noisy environment is considered. The first methods are based on the autocorrelation function (second order cumulants) of the observed sequences. Thus, the autocorrelation sequence is insensitive to the phase characteristics of the system, and a non minimum phase system cannot be identified correctly using the second order statistics [2], their performances degrade when the output is noisy, because the second order cumulants for a gaussian process are not identically zero. To overcome these problems, other approaches were proposed and consist in using higher order cumulants (HOC) [1], this is because the gaussian noise will vanish in the higher order cumulants ( $3^{\text {rd }}$ and $4^{\text {th }}$ cumulants) domain, and the HOC preserve the phase characteristics, unlike the autocorrelation function. Further, the autocorrelation sequence fails to provide a complete statistical description for a non gaussian process, it was shown that consistent estimates of the parameters of any finite impulse response (FIR) system can be obtained by using higher order statistics or cumulants of the observed process [2-4]. Indeed, the HOS constitute a powerful tool in modelling stationary processes when the output signal of a system is corrupte with an additive gaussian noise. However, blind algorithm allows identification of non minimum phase channel with only output measurements, unlike the adaptive

M. Zidane, M. Sabri are with Department of Physics, Faculty of Sciences and technology, Morocco. (e-mail: zidane.ilco@gmail.com; sipt03@yahoo.fr)

S. Safi is with the Department of Mathematics and Informatics, Polydisciplinary Faculty, Morocco. (e-mail: safi.said@gmail.com)

A. Boumezzough is with Department of Physics, Polydisciplinary Faculty, Morocco. (e-mail: ahmed.boumezzough@gmail.com)

Manuscript received August 18, 2015; revised October 4, 2015. algorithms exploiting the information of input and output for the impulse response channel estimation, for this reason the users periodically transmit a training sequence known by the receiver, which then estimates the impulse response channel parameters. Indeed, $20 \%$ of the bandwidth is devoted to training in GSM (up to $40 \%$ in UMTS). Blind methods are thus attractive so as to guarantee a high communication rate by eliminating (or reducing) the training sets [19]. In this paper, we address the problem of the blind and adaptive identification of non minimum phase channel. In order to evaluate the presented algorithms, we consider the Macchi channel model. The simulation results in noisy environment and for different data input channel, demonstrate that the blind and adaptive algorithms could estimate the phase and magnitude of Macchi channel with different precision.

The rest of this paper is organised as follows: In section II, the model and its assumptions, basic relationships are presented. In the following, blind and adaptive algorithms are presented to estimate the parameters of NMP channel. The simulations results showing the performance of the developed methods are presented in section VII. This paper is finally concluded in section VIII.

\section{PROBLEM STATEMENT}

\section{A. Channel modeling}

We consider non minimum phase channel (Fig. 1) described by the following diagram:

In noise free case:

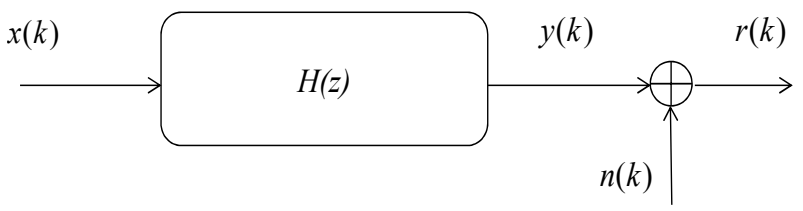

Fig. 1. Channel model

$$
y(k)=\sum_{i=0}^{q} x(i) h(k-i)
$$

In presence of noise:

$$
r(k)=y(k)+n(k),
$$

where $x(i)$ is the input sequence, $h(k)$ and $q$ are the parameters and the order of linear channel, respectively, $y(k)$ represents the channel output in noiseless case and $r(k)$ is the observed channel output corrupted by additive gaussian 
noise $n(k)$.

For this system we assume that:

- The input sequence, $x(i)$, is independent and identically distributed (i.i.d) zero mean, and non-gaussian;

- The system is supposed causal and truncated, i.e. $h(k)=$ 0 for $k<0$ and $k>q$, where $h(0)=1$;

- The system order $q$ is known;

- The measurement noise sequence $n(k)$ is assumed zero mean, i.i.d, gaussian and independent of $x(i)$ with unknown variance.

\section{B. Basic Relationships}

In this section, we present the general relationships for impulse response coefficients that constitute the basic relations in development of most linear HOC based methods proposed in the literature.

The $m^{t h}$ order cumulants of the $y(k)$ can be expressed as a function of impulse response coefficients $h(k)$ as follows [17]:

$$
C_{m, y}\left(t_{1}, t_{2}, \ldots, t_{m-1}\right)=\xi_{m, x} \sum_{i=0}^{q} h(i) h\left(i+t_{1}\right) \ldots h\left(i+t_{m-1}\right),
$$

where $\xi_{m, x}$ represents the $m^{t h}$ order cumulants of the excitation signal $x(i)$ at origin.

If we take $m=2$ into Eq. (3) we obtain the second order cumulant (autocorrelation function):

$$
C_{2, y}\left(t_{1}\right)=\xi_{2, x} \sum_{i=0}^{q} h(i) h\left(i+t_{1}\right)
$$

For $m=3$, Eq. (3) becomes:

$$
C_{3, y}(t)=\xi_{3, x} \sum_{i=0}^{q} h(i) h\left(i+t_{1}\right) h\left(i+t_{2}\right)
$$

Stogioglou and McLaughlin [18] presents the relationship between different $n^{\text {th }}$ cumulants slices of the output signal $y(k)$, and the coefficients $h(k)$, are linked by the following relationship:

$$
\begin{aligned}
& \sum_{j=0}^{q} h(j)\left[\prod_{k=1}^{r} h\left(j+t_{k}\right)\right] C_{n, y}\left(\beta_{1}, \ldots, \beta_{r}, j+\alpha_{1}, \ldots, j+\alpha_{n-r-1}\right) \\
& =\sum_{i=0}^{q} h(i)\left[\prod_{k=1}^{r} h\left(i+\beta_{k}\right)\right] C_{n, y}\left(t_{1}, \ldots, t_{r}, i+\alpha_{1} \ldots, i+\alpha_{n-r-1}\right),
\end{aligned}
$$

where $1 \leq r \leq n-2$.

If we take $n=4$ into Eq. (6) we obtain the following equation:

$$
\begin{aligned}
& \sum_{j=0}^{q} h(j) h\left(j+t_{1}\right) h\left(j+t_{2}\right) C_{4, y}\left(\beta_{1}, \beta_{2}, j+\alpha_{1}\right) \\
& =\sum_{i=0}^{q} h(i) h\left(i+\beta_{1}\right) h\left(i+\beta_{2}\right) C_{4, y}\left(t_{1}, t_{2}, i+\alpha_{1}\right)
\end{aligned}
$$

\section{SAFI, et al. Algorithm : ALGcum 1}

The Fourier transform of Eqs. (4) and (5) gives us the bispectra and the spectrum respectively [5]:

$$
S_{3, y}\left(\omega_{1}, \omega_{2}\right)=\xi_{3, x} H\left(-\omega_{1}-\omega_{2}\right) H\left(\omega_{1}\right) H\left(\omega_{2}\right)
$$

$$
S_{2, y}(\omega)=\xi_{2, x} H(-\omega) H(\omega)
$$

If we suppose that $\omega=\omega_{1}+\omega_{2}$, Eq. (9) becomes:

$$
S_{2, y}\left(\omega_{1}+\omega_{2}\right)=\xi_{2, x} H\left(-\omega_{1}-\omega_{2}\right) H\left(\omega_{1}+\omega_{2}\right)
$$

Then, from Eqs. (8) and (10) we obtain the following equation:

$$
S_{3, y}\left(\omega_{1}, \omega_{2}\right) H\left(\omega_{1}+\omega_{2}\right)=\mu H\left(\omega_{1}\right) H\left(\omega_{2}\right) S_{2, y}\left(\omega_{1}+\omega_{2}\right),
$$

with $\mu=\frac{\xi_{3, x}}{\xi_{2, x}}$

The inverse Fourier transform of Eq. (11) demonstrates that the $3^{\text {rd }}$ order cumulants, the autocorrelation function and the impulse response channel parameters are combined by the following equation [5]:

$\sum_{i=0}^{q} C_{3, y}\left(t_{1}-i, t_{2}-i\right) h(i)=\mu \sum_{i=0}^{q} h(i) h\left(t_{2}-t_{1}+i\right) C_{2, y}\left(t_{1}-i\right)$

If we use the autocorrelation function property of the stationary process such as $C_{2, y}(t) \neq 0$ only for $-q \leq t \leq q$ and vanishes elsewhere if we take $t_{1}=-q$, Eq. (12) takes the forme [5]:

$$
\sum_{i=0}^{q} C_{3, y}\left(-q-i, t_{2}-i\right) h(i)=\mu h(0) h\left(t_{2}+q\right) C_{2, y}(-q),
$$

else, if we suppose that $t_{2}=-q$, Eq. (13) will become:

$$
C_{3, y}(-q,-q) h(q)=\mu h(0) C_{2, y}(-q)
$$

Using Eqs. (13) and (14) we obtain the following relation [5]:

$$
\sum_{i=0}^{q} C_{3, y}\left(-q-i, t_{2}-i\right) h(i)=C_{3, y}(-q,-q) h\left(t_{2}+q\right)
$$

The system of Eq. (15) can be written in matrix form as:

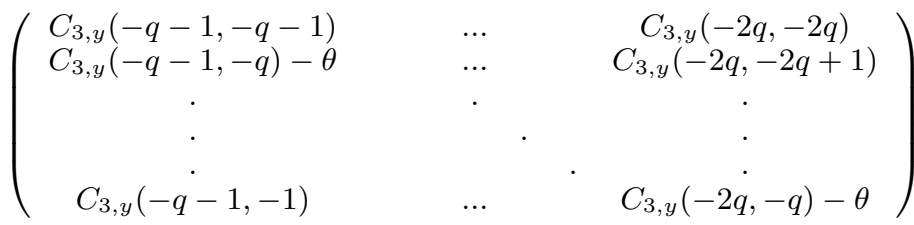

$$
\begin{aligned}
& \times\left(\begin{array}{c}
h(1) \\
\cdot \\
\cdot \\
\dot{h(i)} \\
\cdot \\
\cdot \\
\cdot \dot{h(q)}
\end{array}\right)=\left(\begin{array}{c}
0 \\
-C_{3, y}(-q,-q+1) \\
\cdot \\
\cdot \\
\cdot \\
\left.-C_{3, y} \dot{(}-q, 0\right)
\end{array}\right) \text {, }
\end{aligned}
$$

where $\theta=C_{3, y}(-q,-q)$.

Or in more compact form, the Eq. (16) can be written as follows:

$$
M h_{q}=d,
$$

where $M$ is the matrix of size $(q+1) \times(q)$ elements, $h_{q}$ is a column vector constituted by the unknown impulse response parameters $h(k)$ for $k=1, \ldots, q$ and $d$ is a column vector of size $(q+1)$ as indicated in the Eq. (17). 
The least squares solution of the system of Eq. (17), permits blindly identification of the parameters $h(k)$ and without any information of the input selective channel. Thus, the solution will be written under the following form:

$$
\widehat{h}_{q}=\left(M^{T} M\right)^{-1} M^{T} d
$$

\section{Antari, et al. Algorithm : ALGcum2}

Antari, et al. [9] demonstrates that the coefficients $h(k)$ for an finite impulse response system can be obtained by the Eq. (12), if we take $t_{2}-t_{1}=q$ and the considered system is causal. The following equation describes (ALGcum2) algorithm:

$$
\sum_{i=0}^{q} C_{3, y}\left(t_{1}-i, t_{1}+q-i\right) h(i)=\frac{\xi_{3, x}}{\xi_{2, x}} h(0) h(q) C_{2, y}\left(t_{1}\right),
$$

where $t_{1}=-q, \ldots, q$ the Eq. (19) can be written as follows:

$$
M h_{e}=d
$$

where $M$ is the matrix of size $(2 q+1) \times(q+1)$ elements, $h_{e}$ is a column vector constituted by the unknown impulse response parameters $h(k): k=0, \ldots, q$ and $d$ is a column vector of size $(2 q+1)$ as indicated in the Eq. (20). The least squares solution of the system of Eq. (20), will be written under the following form:

$$
\widehat{h}_{e}=\left(M^{T} M\right)^{-1} M^{T} d
$$

\section{Abderrahim, et al. Algorithm : ALGcum3}

Abderrahim, et al. [10] use the relationship (7), based on fourth order cumulants, with $\beta_{1}=\beta_{2}=0, t_{1}=q$ and $t_{2}=0$. This algorithm is given by the following relation:

$\sum_{i=0}^{q} h^{3}(i) C_{4, y}\left(q, 0, i+\alpha_{1}\right)-h(q) C_{4, y}\left(0,0, \alpha_{1}\right)=-C_{4, y}\left(q, 0, \alpha_{1}\right)$

where $\alpha_{1}=-q, \ldots, q$. In more compact form, the system of Eq. (22) can be written in the following form:

$$
M \theta=A
$$

$\theta=\left[h(q) \quad h^{3}(1) \quad h^{3}(2) \ldots . h^{3}(q)\right]^{T}$ is a column vector of size $(q+1)$;

$A=\left[0 \ldots 0-C_{4 y}(q, 0,0)-C_{4 y}(q, 0,1) \ldots-C_{4 y}(q, 0, q)\right]^{T}$ is a vector of size $(2 q+1)$;

The least squares solution of the system of Eq. (23), will be written under the following form:

$$
\widehat{\theta}=\left(M^{T} M\right)^{-1} M^{T} d
$$

The parameters $h(i)$ for $k=1, \ldots, q$ are estimated from the estimated values $\widehat{\theta}(i)$ using the following equation:

$$
\widehat{h}(i)=\sqrt[3]{\widehat{\theta}(i+1)}
$$

\section{ADAPTIVE ALGORITHMS}

\section{A. Description of the LMS Algorithm}

From the method of steepest descent, the weight vector equation is given by [20]:

$$
\widehat{H}_{n+1}=\widehat{H}_{n}-\left.\frac{1}{2} \mu \nabla \bar{\varepsilon}(H)\right|_{\widehat{H}_{n}},
$$

with $e(n)=r(n)-X^{T}(n) H, \bar{\varepsilon}=E\left[|e(n)|^{2}\right]$ and $H=$ $[h(0), h(1), \ldots, h(q)]^{T} . \mu$ is the step-size parameter and controls the convergence characteristics of the LMS algorithm including between 0 and 1 .

The gradient vector in the above weight update equation can be computed as:

$$
\begin{gathered}
\left.\nabla \bar{\varepsilon}(H)\right|_{\widehat{H}_{n}} \approx \nabla|e(n)|^{2}=\left.\frac{\partial|e(n)|^{2}}{\partial H}\right|_{\widehat{H}_{n}} \\
\nabla|e(n)|^{2}=-2 e(n) X(n)
\end{gathered}
$$

The LMS algorithm is initiated with an arbitrary value $h(0)$ for the weight vector at $n=0$. The successive corrections of the weight vector eventually leads to the minimum value of the mean squared error. Therefore the LMS algorithm can be summarized in the following equations:

$$
\widehat{H}(n+1)=\widehat{H}(n)+\mu \widehat{e}(n) X(n),
$$

with

$$
\widehat{e}(n)=r(n)-\widehat{H}_{n} X^{T}(n)
$$

\section{B. Description of the RLS Algorithm}

The RLS algorithm [12] is discribed by the following equations (with initial conditions $\widehat{H}(0)=0$ ). $Q^{-1}(0)=\delta^{-1} I, \delta$ is a small positive constant value.

$$
\begin{gathered}
k(n)=\frac{\lambda^{-1} Q^{-1}(n-1) X(n)}{1+\lambda^{-1} X^{T}(n) Q^{-1}(n-1) X(n)}, \\
e(n)=r(n)-X^{T}(n) \widehat{H}(n-1), \\
\widehat{H}(n)=\widehat{H}(n-1)+k(n) e(n),
\end{gathered}
$$

$$
Q^{-1}(n)=\lambda^{-1} Q^{-1}(n-1)-\lambda^{-1} k(n) X^{T}(n) Q^{-1}(n-1) .
$$

\section{SIMULATION RESULTS}

To verify the performance of these algorithms, we have applied a Macchi channel model. To measure the strength of noise, we define the signal to noise ratio $(S N R)$ as:

$$
S N R=10 \log \left[\frac{\sigma_{y}^{2}(k)}{\sigma_{n}^{2}(k)}\right]
$$

To measure the accuracy of parameter estimation with respect to the real values, we define the mean square error $(M S E)$ for each run as

$$
M S E=\frac{1}{q} \sum_{i=0}^{q}\left[\frac{h(i)-\widehat{h}(i)}{h(i)}\right]^{2},
$$


where $\widehat{h}(i), i=1, \ldots, q$ are the estimated parameters in each run, and $h(i), i=1, \ldots, q$ are the real parameters in the model.

1) Macchi channel: The Macchi channel is defined by the following equation:

$$
\left\{\begin{array}{c}
y(k)=0.8264 x(k)-0.1653 x(k-1)+0.8512 x(k-2) \\
+0.1636 x(k-3)+0.8100 x(k-4), \\
\text { zeros: } z_{1}=0.5500+0.9526 j ; z_{2}=0.5500-0.9526 j ; \\
z_{3}=-0.4500+0.7794 j ; z_{4}=-0.4500-0.7794 j .
\end{array}\right.
$$

The Macchi channel is a non minimum phase because two of its zeros are outside of the unit circle.

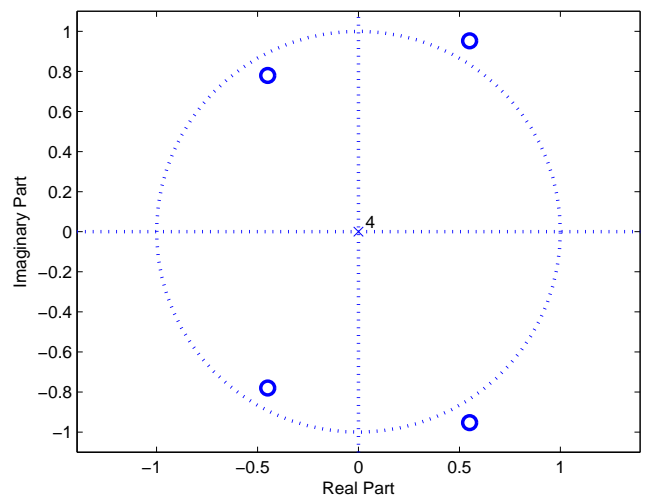

Fig. 2. The zeros of Macchi channel

2) Impulse response parameters estimation: The results of simulation are shown in the Table I for different values of sample sizes and for $\mathrm{SNR}=8 \mathrm{~dB}$.

For all sample sizes and for a $\mathrm{SNR}=8 \mathrm{~dB}$, the values, of mean square error (MSE), of the (ALGcum 1$), L M S$ and $R L S$ algorithms are small than those obtained by the (ALGcum2) and (ALGcum 3$)$ algorithms, this implies the true parameters are near the estimates parameters.

The Fig. 3 give us a good idea about the precision of these algorithms.

In the Table II we well present the impulse response parameters estimation of the Macchi's channel for different $S N R$ and data length $N=2048$.

From the Table II we can conclude that: the $R L S, L M S$ and (ALGcum1) algorithms are more effective than (ALGcum2) and (ALGcum3) algorithms in noisy environment, with an advantage of the $R L S$ algorithm in terms of $M S E$. But the results given by the algorithms based on higher order cumulants, are blindly without any information about the input, comparing to adaptive algorithms exploiting the information of input and output for the estimation of the impulse response channel. For that the adaptive methods send occasionally a training sequence known to the transmitter and receiver.

The Fig. 4 give us a good idea about the precision of these algorithms for different $S N R$.

3) Magnitude and phase estimation: In the following figure (Fig. 5) we have presented the estimation of the magnitude and phase of the impulse response channel using the presented

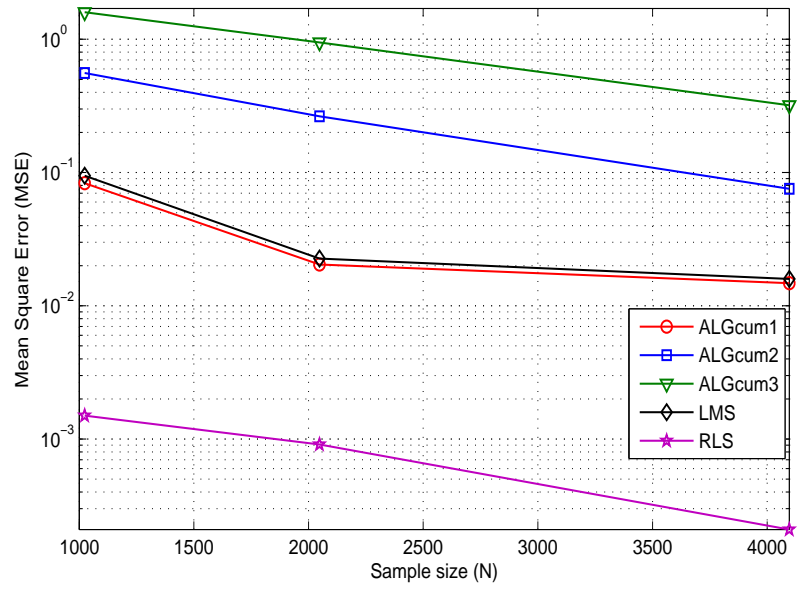

Fig. 3. Comparison of algorithms for Macchi channel for $\mathrm{SNR}=8 \mathrm{~dB}$

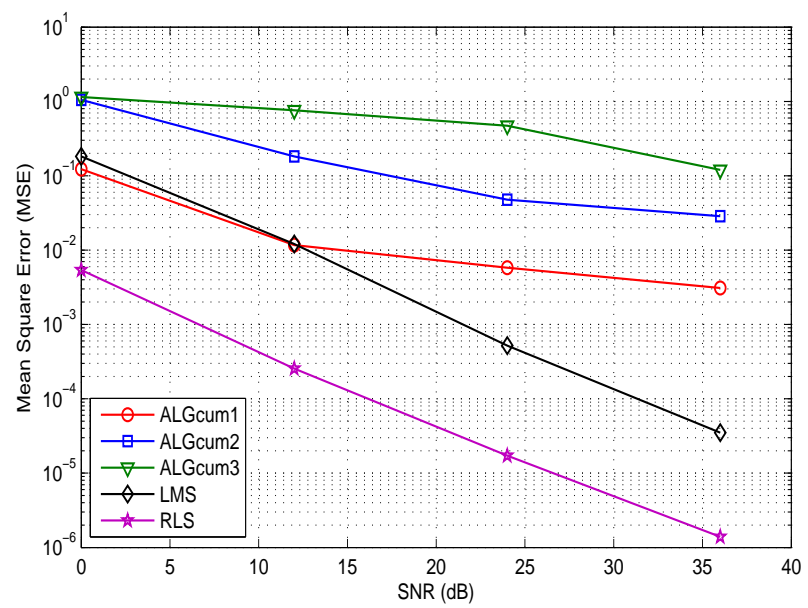

Fig. 4. Comparison of algorithms for Macchi channel for $N=2048$

algorithms for $N=2048$ and $\mathrm{SNR}=8 \mathrm{~dB}$.

From the Fig. 5 we observe that the estimated magnitude and

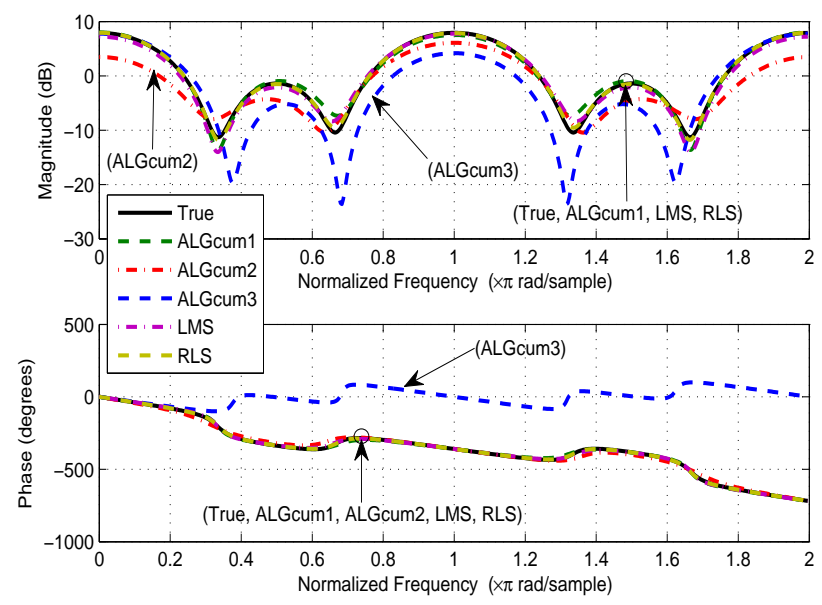

Fig. 5. Estimated magnitude and phase of the Macchi channel impulse response, for different algorithms, $N=2048$ and $\mathrm{SNR}=8 \mathrm{~dB}$. 
TABLE I

Estimated parameters of Macchi channel model excited by different sample sizes and for SNR $=8 \mathrm{~dB}$

\begin{tabular}{|c|c|c|c|c|c|c|}
\hline$N$ & $\widehat{h}(i) \pm \sigma$ & ALGcum 1 & $L M S$ & ALGcum2 & ALGcum3 & $R L S$ \\
\hline \multirow{6}{*}{1024} & $\widehat{h}(1) \pm \sigma$ & $0.7896 \pm 0.1338$ & $0.8456 \pm 0.0629$ & $0.5607 \pm 0.2748$ & $0.5101 \pm 0.5314$ & $0.8114 \pm 0.0070$ \\
\hline & $\widehat{h}(2) \pm \sigma$ & $-0.0559 \pm 0.2056$ & $-0.2734 \pm 0.1140$ & $-0.4244 \pm 0.1628$ & $0.2557 \pm 0.4035$ & $-0.1588 \pm 0.0071$ \\
\hline & $\widehat{h}(3) \pm \sigma$ & $0.7857 \pm 0.1099$ & $0.8075 \pm 0.0874$ & $0.5550 \pm 0.3079$ & $0.5790 \pm 0.6220$ & $0.8536 \pm 0.0060$ \\
\hline & $\widehat{h}(4) \pm \sigma$ & $0.1892 \pm 0.1053$ & $0.1886 \pm 0.0901$ & $0.0302 \pm 0.1063$ & $-0.1049 \pm 0.5160$ & $0.1759 \pm 0.0079$ \\
\hline & $\widehat{h}(5) \pm \sigma$ & $0.6740 \pm 0.1408$ & $0.6982 \pm 0.1735$ & $0.8061 \pm 0.2618$ & $0.4907 \pm 0.9437$ & $0.8076 \pm 0.0069$ \\
\hline & $M S E$ & 0.0831 & 0.0946 & 0.5577 & 1.5976 & 0.0015 \\
\hline \multirow{6}{*}{2048} & $\widehat{\widehat{h}(1) \pm \sigma}$ & $0.8797 \pm 0.1168$ & $\overline{0.8233 \pm 0.0662}$ & $\overline{0.5137 \pm 0.1847}$ & $\overline{0.6921 \pm 0.3818}$ & $0.8351 \pm 0.0048$ \\
\hline & $\widehat{h}(2) \pm \sigma$ & $-0.1506 \pm 0.1482$ & $-0.2039 \pm 0.0512$ & $-0.3257 \pm 0.0969$ & $0.2236 \pm 0.3769$ & $-0.1643 \pm 0.0042$ \\
\hline & $\widehat{h}(3) \pm \sigma$ & $0.8154 \pm 0.1087$ & $0.8306 \pm 0.0510$ & $0.6500 \pm 0.2366$ & $0.7497 \pm 0.4089$ & $0.8565 \pm 0.0067$ \\
\hline & $\widehat{h}(4) \pm \sigma$ & $0.2161 \pm 0.0830$ & $0.1290 \pm 0.0557$ & $0.0642 \pm 0.1143$ & $0.1903 \pm 0.4751$ & $0.1737 \pm 0.0054$ \\
\hline & $\widehat{h}(5) \pm \sigma$ & $0.7499 \pm 0.1224$ & $0.7181 \pm 0.0506$ & $0.5890 \pm 0.2197$ & $0.5895 \pm 0.4283$ & $0.7905 \pm 0.0063$ \\
\hline & $M S E$ & 0.0204 & 0.0226 & 0.2641 & 0.9459 & $9.1098 \times 10^{-4}$ \\
\hline \multirow{7}{*}{4096} & $\widehat{h}(1) \pm \sigma$ & $0.7871 \pm 0.0805$ & $0.8185 \pm 0.0297$ & $0.5806 \pm 0.1338$ & $0.7450 \pm 0.1372$ & $0.8287 \pm 0.0050$ \\
\hline & $\widehat{h}(2) \pm \sigma$ & $-0.1977 \pm 0.1038$ & $-0.1198 \pm 0.0236$ & $-0.2438 \pm 0.0730$ & $0.0105 \pm 0.3597$ & $-0.1625 \pm 0.0041$ \\
\hline & $\widehat{h}(3) \pm \sigma$ & $0.8552 \pm 0.0740$ & $0.8569 \pm 0.0277$ & $0.6203 \pm 0.1822$ & $0.7980 \pm 0.1381$ & $0.8526 \pm 0.0041$ \\
\hline & $\widehat{h}(4) \pm \sigma$ & $0.1281 \pm 0.0526$ & $0.1601 \pm 0.0304$ & $0.1821 \pm 0.1147$ & $0.0299 \pm 0.5077$ & $0.1681 \pm 0.0050$ \\
\hline & $\widehat{h}(5) \pm \sigma$ & $0.8335 \pm 0.0877$ & $0.8568 \pm 0.0296$ & $0.6233 \pm 0.1701$ & $0.5475 \pm 0.2014$ & $0.8106 \pm 0.0043$ \\
\hline & $M S E$ & 0.0148 & 0.0159 & 0.0756 & 0.3197 & $2.0973 \times 10^{-4}$ \\
\hline & True parameters $h(i)$ & $\bar{h}(1)=0.8264$ & $h(2)=-0.1653$ & $\overline{h(3)=0.8512}$ & $\overline{~ h(4)=0.1636}$ & $h(5)=0.8100$ \\
\hline
\end{tabular}

TABLE II

Estimated parameters of Macchi channel model for different $S N R$ and excited by sample sizes $N=2048$

\begin{tabular}{|c|c|c|c|c|c|c|}
\hline$S N R$ & $\widehat{h}(i) \pm \sigma$ & ALGcum 1 & $L M S$ & ALGcum2 & ALGcum 3 & $R L S$ \\
\hline \multirow{6}{*}{$0 \mathrm{~dB}$} & $\widehat{h}(1) \pm \sigma$ & $0.8528 \pm 0.1256$ & $0.8309 \pm 0.0540$ & $-0.1310 \pm 0.9704$ & $-0.0146 \pm 0.5764$ & $0.8446 \pm 0.0127$ \\
\hline & $\widehat{h}(2) \pm \sigma$ & $-0.1157 \pm 0.1140$ & $-0.2864 \pm 0.0839$ & $-0.1543 \pm 0.5630$ & $0.0316 \pm 0.4848$ & $-0.1660 \pm 0.0148$ \\
\hline & $\widehat{h}(3) \pm \sigma$ & $0.7554 \pm 0.1463$ & $0.8615 \pm 0.0602$ & $0.1448 \pm 0.8429$ & $0.2827 \pm 0.6526$ & $0.8624 \pm 0.0152$ \\
\hline & $\widehat{h}(4) \pm \sigma$ & $0.2935 \pm 0.0777$ & $0.0700 \pm 0.0455$ & $-0.1602 \pm 0.2333$ & $-0.1418 \pm 0.4816$ & $0.1882 \pm 0.0147$ \\
\hline & $\widehat{h}(5) \pm \sigma$ & $0.7634 \pm 0.1416$ & $0.6345 \pm 0.0537$ & $0.3275 \pm 0.6321$ & $0.2376 \pm 0.5866$ & $0.7607 \pm 0.0125$ \\
\hline & $M S E$ & 0.1229 & 0.1823 & 1.0514 & 1.1477 & 0.0054 \\
\hline \multirow{6}{*}{$12 \mathrm{~dB}$} & $\widehat{h}(1) \pm \sigma$ & $0.8752 \pm 0.1081$ & $0.8294 \pm 0.0167$ & $0.5806 \pm 0.1957$ & $0.7387 \pm 0.1427$ & $0.8317 \pm 0.0037$ \\
\hline & $\widehat{h}(2) \pm \sigma$ & $-0.1938 \pm 0.1234$ & $-0.1940 \pm 0.0128$ & $-0.3110 \pm 0.1091$ & $0.0957 \pm 0.3542$ & $-0.1656 \pm 0.0036$ \\
\hline & $\widehat{h}(3) \pm \sigma$ & $0.8311 \pm 0.1096$ & $0.8412 \pm 0.0147$ & $0.6797 \pm 0.1813$ & $0.8193 \pm 0.1271$ & $0.8548 \pm 0.0044$ \\
\hline & $\widehat{h}(4) \pm \sigma$ & $0.1942 \pm 0.0583$ & $0.1363 \pm 0.0115$ & $0.1062 \pm 0.1204$ & $-0.0655 \pm 0.5145$ & $0.1687 \pm 0.0048$ \\
\hline & $\widehat{h}(5) \pm \sigma$ & $0.7810 \pm 0.1101$ & $0.7721 \pm 0.0159$ & $0.6071 \pm 0.1848$ & $0.5510 \pm 0.2510$ & $0.7975 \pm 0.0036$ \\
\hline & $M S E$ & 0.0117 & 0.0121 & 0.1819 & 0.7615 & $2.5485 \times 10^{-4}$ \\
\hline \multirow{6}{*}{$24 \mathrm{~dB}$} & $\widehat{h}(1) \pm \sigma$ & $0.8534 \pm 0.0920$ & $0.8256 \pm 0.0037$ & $0.7273 \pm 0.1873$ & $0.8027 \pm 0.1540$ & $0.8277 \pm 0.0008$ \\
\hline & $\widehat{h}(2) \pm \sigma$ & $-0.1814 \pm 0.1497$ & $-0.1703 \pm 0.0038$ & $-0.2460 \pm 0.0979$ & $0.0862 \pm 0.4024$ & $-0.1654 \pm 0.0011$ \\
\hline & $\widehat{h}(3) \pm \sigma$ & $0.8767 \pm 0.0989$ & $0.8517 \pm 0.0047$ & $0.8098 \pm 0.1859$ & $0.8242 \pm 0.1691$ & $0.8519 \pm 0.0008$ \\
\hline & $\widehat{h}(4) \pm \sigma$ & $0.1400 \pm 0.0942$ & $0.1572 \pm 0.0039$ & $0.1914 \pm 0.1294$ & $0.0515 \pm 0.5461$ & $0.1649 \pm 0.0008$ \\
\hline & $\widehat{h}(5) \pm \sigma$ & $0.8486 \pm 0.1029$ & $0.7994 \pm 0.0038$ & $0.7681 \pm 0.2359$ & $0.6482 \pm 0.3391$ & $0.8067 \pm 0.0009$ \\
\hline & $M S E$ & $58 \times 10^{-4}$ & $5.1989 \times 10^{-4}$ & 0.0478 & 0.4710 & $1.7192 \times 10^{-5}$ \\
\hline \multirow{7}{*}{$36 \mathrm{~dB}$} & $\widehat{h}(1) \pm \sigma$ & $0.8564 \pm 0.0982$ & $0.8267 \pm 0.0012$ & $0.7103 \pm 0.1974$ & $0.8383 \pm 0.1537$ & $0.8268 \pm 0.0002$ \\
\hline & $\widehat{h}(2) \pm \sigma$ & $-0.1728 \pm 0.1327$ & $-0.1667 \pm 0.0008$ & $-0.2233 \pm 0.0886$ & $-0.0428 \pm 0.3878$ & $-0.1653 \pm 0.0003$ \\
\hline & $\widehat{h}(3) \pm \sigma$ & $0.8887 \pm 0.0986$ & $0.8509 \pm 0.0010$ & $0.7805 \pm 0.2284$ & $0.8728 \pm 0.1253$ & $0.8514 \pm 0.0003$ \\
\hline & $\widehat{h}(4) \pm \sigma$ & $0.1552 \pm 0.0733$ & $0.1620 \pm 0.0009$ & $0.1872 \pm 0.1490$ & $0.2287 \pm 0.5115$ & $0.1640 \pm 0.0002$ \\
\hline & $\widehat{h}(5) \pm \sigma$ & $0.8940 \pm 0.0958$ & $0.8079 \pm 0.0009$ & $0.7859 \pm 0.2864$ & $0.7197 \pm 0.3714$ & $0.8092 \pm 0.0002$ \\
\hline & $M S E$ & $31 \times 10^{-4}$ & $3.5059 \times 10^{-5}$ & 0.0286 & 0.1201 & $1.3921 \times 10^{-6}$ \\
\hline & True parameters $h(i)$ & $h(1)=0.8264$ & $h(2)=-0.1653$ & $\overline{~ h(3)=0.8512}$ & $h(4)=0.1636$ & $\bar{~} h(5)=0.8100$ \\
\hline
\end{tabular}

phase using (ALGcum1), LMS and $R L S$ algorithms have the same allure and we have not more difference between the estimated and the true ones. The (ALGcum2) algorithm is able to estimate the phase response, but we have more difference between the magnitude estimated and the true ones, of the Macchi channel. The results given by (ALGcum 3 ) algorithm are more different to the true ones.

\section{CONCLUSION}

In this paper, we have compared blind identification methods based on HOC, with the adaptive algorithms ( $R L S$ and $L M S$ ), to identify the parameters of the impulse response of the frequency selective channel such as the Macchi channel. The simulation results show the efficiency of the $R L S$, $L M S$ and (ALGcum 1 ) algorithms, but the blind algorithm present the advantage to estimate the impulse response of the frequency selective channel blindly, comparing to adaptive algorithms. The magnitude and phase of the impulse response are estimated, using $R L S, L M S$ and (ALGcum1) algorithms with a very good precision in noisy environment, but the (ALGcum2) and (ALGcum 3 ) give us a significant difference between the estimated and the true ones. 


\section{REFERENCES}

[1] K. Abederrahim, H. Mathlouthi and F. Msahli, "FIR System Identification using Higher Order Statistics," American Control Conference Hyatt Regency Riverfront, St. Louis, MO, USA June 10-12, pp. 5598-5603, 2009.

[2] L. Srinivas and K. V. S. Hari, "FIR System Identification Based on Subspaces of a Hight Order Cumulant Matrix," IEEE Transactions on signal processing, vol. 44 no. 6, pp. 1485-1491, 1996.

[3] M. Bakrim and D. Aboutajdine, "Cumulant based identification of non gaussian moving average signals," Traitement du Signal, vol. 16 no. 3 pp. $175-186,1999$.

[4] C.-Y. Chi and J.-Y. Kung, "A new identification algorithm for all pass systems by higher order statistics," Signal Processing vol. 41, pp. 239-256, 1995.

[5] S. Safi, M. Frikel, M. M'Saad, and A. Zeroual, "Blind Impulse Response Identification of frequency Radio Channels : Application to Bran A Channel," Int. J. Sig. Proces, vol. 4 no. 1 pp. 201-206, 2007.

[6] S. Safi and A. Zeroual, "Blind non minimum phase channel identification using $3^{\text {rd }}$ and $4^{\text {th }}$ order cumulants," Int. J. Sig. Proces., vol. 4 no. 1, pp. $158-168,2008$.

[7] M. Zidane, S. Safi, M. Sabri and A. Boumezzough, "Impulse Response Identification of Minimum and Non Minimum Phase Channels," $4^{\text {th }}$ Workshop on Codes, Cryptography and Communication Systems (WCCCS'13) 07-08 November 2013, Meknes, Morocco.

[8] J. Antari, A. El Khadimi, D. Mammas and A. Zeroual, "Developed Algorithm for Supervising Identification of Non Linear Systems using Higher Order Statistics: Modeling Internet Traffic," International Journal of Future Generation Communication and Networking, vol. 5 no. 4, pp. $17-28,2012$.

[9] J. Antari, A. Zeroual and S. Safi, "Stochastic analysis and parametric identification of moving average (MA) non Gaussian signal using cumulants," International Journal of Physical and Chemical News, vol. 34, pp. 27-32, 2007.

[10] K. Abderrahim, R. B. Abdennour, F. Msahli, M. Ksouri, and G. Favier "Identification of non minimum phase finite impulse response systems using the fourth order cumulants," Progress in system and robot analysis and control design, Springer, vol. 243, pp. 41-50, 1999.

[11] K. Abderrahim, R. B. Abdennour, G. favier, M. Ksouri and F Msahli, "New results on FIR system identification using cumulants," APII-JESA., vol. 35 no 5, pp. 601-622, 2001.

[12] A. Y. Kibangou, "Modèles de volterra à complexitè reduite : estimation parametrique et application à l'égalisation des canaux de communication," Thèse de doctorat, Universit de Nice-Sophia Antipolis, 2005.

[13] M. Zidane, S. Safi, M. Sabri, and A. Boumezzough, "Blind Identification of Minimum Phase Channels Based On Higher Order Cumulants," International Conference on Intelligent Information and Network Technology (IC2INT'13) 13-14 November 2013, Settat, Morocco.

[14] S. Safi, A. Zeroual and M. M. Hassani, "Prediction of global daily solar radiation using higher order statistics," Renewable Energy, vol. 27, pp. 647-666, 2002.

[15] S. Safi and A. Zeroual, "Blind identification in noisy environment of non minimum phase Finite Impulse Response (FIR) using higher order statistics," Int. J. Sys. Anal. Modell. Simul., Taylor Francis, vol. 43 no 5, pp. 671-681, 2003.

[16] J. Antari, R. Iqdour and A. Zeroual, "Forecasting the wind speed process using higher order statistics and fuzzy systems," Renewable Energy, vol. $9,237-251,2006$

[17] D. Brillinger and M. Rosenblatt, "Computation and interpretation of $k^{\text {th }}$ order spectra," In Spectral Analysis of Time Signals, NewYork: Wiley, pp. $907-938,1967$.

[18] A. G. Stogioglou and S. McLaughlin, "MA parameter estimation and cumulant enhancement," IEEE Transactions on Signal Processing, vol. 44 no. 7, pp. 1704-1718, 1996.

[19] D. Nion and L. De Lathauwer, "A Block Component Model Based Blind DS-CDMA Receiver," IEEE Transactions on signal processing, vol. 56 no. 11 , pp. 5567-5579, 2008.

[20] T. Aboulnasr and K. Mayyas, "A robust variable step-size LMS-type algorithm : analysis and simulations," IEEE Transactions on Signal Processing, vol. 45 no. 3, pp. 631-639, 1997. 